\title{
Endoscopic ultrasound-guided fine-needle aspiration (EUS-FNA) of paraesophageal lung tumors - diagnostic yield and added value
}

\author{
Romeo Ioan Chira ${ }^{1}$, Alexandra Chira ${ }^{2}$, Vlad Andrei Ichim ${ }^{1}$, Nagy Anca Georgiana ${ }^{1}$, \\ Alina Florea ${ }^{3}$, Doiniţa Crişan ${ }^{4}$, Bogdan Popovici $^{5}$
}

${ }^{1} 1^{\text {st }}$ Medical Clinic, Div. Gastroenterology, Emergency Clinical County Hospital Cluj-Napoca; Department of Internal Medicine, "Iuliu Hatieganu" University of Medicine and Pharmacy, ${ }^{2}{ }^{\text {nd }}$ Medical Clinic, Department of Internal

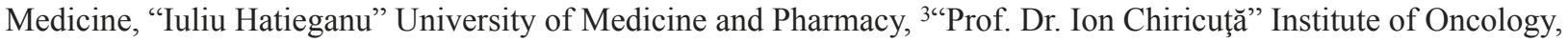
${ }^{4}$ Pathology Department, Emergency Clinical County Hospital Cluj, Cluj-Napoca, Romania, Department of Morphological Sciences, "Iuliu Hatieganu” University of Medicine and Pharmacy, ${ }^{5}$ Department of Thoracic Surgery, "Leon Danilello" Clinical Hospital of Pneumology, Cluj-Napoca, Romania

\begin{abstract}
Aims: There are few data on the use of endoscopic ultrasound (EUS) for the biopsy of suspected malignant lesions of the lung. The main objective of this study was to evaluate the performance of transesophageal EUS fine needle aspiration (EUSFNA) for the diagnosis of paraesophageal lung tumors and also for the confirmation of metastatic sites of lung cancer during the same procedure. Material and methods: We performed a retrospective study in a tertiary care unit including 19 patients with paraesophageal lung tumors referred to our department for a lung biopsy. Transesophageal EUS-FNA was performed using a linear echoendoscope and 22G needles. Results: In all 19 patients with suspected lung tumors the confirmation of the malignant disease was achieved. Pathological examination revealed 16 cases of non-small cell lung cancers, 2 small cell lung cancers and one case of lung metastases. Diagnostic yield of lung EUS-FNA was 1, with no post-procedural complications. In 7 cases, we performed also biopsies of suspected metastasis and all biopsies revealed the same histopathological type as the primary tumor. Conclusions: Our study supports the use of this minimally invasive technique for paraesophageally located lung tumors and demonstrates that EUS-FNA is safe and has an excellent diagnostic yield.
\end{abstract}

Keywords: endoscopic ultrasound-fine needle aspiration (EUS-FNA); lung; cancer; biopsy

\section{Introduction}

Lung cancer represents an important health problem worldwide, causing the most frequent malignancy related death [1]. Diagnosis of lung cancer is frequently sug-

Received 02.09.2019 Accepted 30.10.2019

Med Ultrason

2019, Vol. 21, No 4, 377-381

Corresponding author: Alexandra Chira, MD, PhD

$2^{\text {nd }}$ Medical Clinic, 2-4 Clinicilor Street,

400006, Cluj-Napoca, Romania

Phone: +40721249635

E-mail: Chira.Alexandra@umfcluj.ro gested by radiological techniques, especially computed tomography (CT), but a personalized management needs precise pathological diagnosis. This can be obtained through tissue biopsy via either transluminal, percutaneous or surgical approach. When tumors are in contact or near to the major airways, endobronchial ultrasound (EBUS) can provide a histological diagnosis. For centrally located lung tumors, without endobronchial abnormalities or in close relation to the airways, endobronchial techniques are rarely efficient and fail to establish the diagnosis [2].

Centrally located lung tumors can be biopsied under CT guidance, but the sensitivity is lower than for 
the peripheral tumors and the complication rate is much higher than for endoscopic ultrasound (EUS) fine needle aspiration (EUS-FNA) [3-5]. The pneumothorax incidence after CT guided biopsy was reported to be $9-54 \%$ $[3,6,7]$. The small diameter of the paramediastinal lung tumors (less than $2 \mathrm{~cm}$ ) also decreases the sensitivity of CT guided biopsy [8]. These issues brought EUS to the forefront, in order to provide a minimally invasive diagnosis in these cases. The procedure is indicated when the lung tumor has a close relation with the esophagus or suspicious mediastinal lymph nodes can be visualized and punctured through a transesophageal approach $[4,9,10]$.

The importance of EUS for mediastinal lymph nodes evaluation and lung cancer staging is recognized and supported by European guidelines [4]. But the literature concerning the transesophageal biopsy of the lung cancer is still sparse, and in many medical centers this procedure is not performed $[11,12]$. There are very few studies including patients with previous non-diagnostic bronchoscopy that underwent EUS-FNA addressed to paraesophageal lung tumors $[9,10]$ and few lung EUSFNA studies included patients having either a negative bronchoscopy or a negative CT-guided biopsy [13-16]. A recent study supports the added value of EUS-FNA after a non-diagnostic bronchoscopy, showing an increase of the diagnostic yield from 51 to $91 \%$ [17].

Another issue concerning the added value of EUSFNA in lung cancer staging is the possibility to procure samples also from extra thoracic suspected metastatic lesions - from the liver, adrenal glands, retroperitoneal lymph nodes etc $[18,19]$.

We conducted this study in order to assess the sensitivity and diagnostic yield of EUS-FNA in paraesophageal lung tumors in patients with negative bronchoscopy and to evaluate the feasibility of the method to confirm the metastatic sites of the lung cancer at the same session.

\section{Material and methods}

\section{Study design and patients}

This was a monocentric retrospective study of the chart files, undertaken at the Emergency County Clinical Hospital Cluj-Napoca, Department of Gastroenterology and the Iuliu Hatieganu University of Medicine and Pharmacy of Cluj-Napoca, between 1 July 2016 and 1 July 2019. The study was approved by the Ethics Committee of the University of Medicine and Pharmacy.

We selected for inclusion consecutive patients who had underwent a EUS-FNA in order to obtain a tissue specimen from an intrapulmonary lesion suspected to be lung cancer, patients in which the routinely performed bronchoscopy was non-diagnostic. All the patients se- lected had lung tumors located paraesophageally. The patients with thoracic CT scans showing thoracic aorta interposition between the tumor and the esophagus and those diagnosed by surgical biopsy were excluded. All CT and PET-CT imaging, bronchoscopy, EBUS and EUS reports, histopathological and follow-up data were collected.

Patients were followed up for complications during admission for at least $24 \mathrm{~h}$ and as outpatients for 30 days.

The primary endpoint of the study was to assess the diagnostic yield of EUS-FNA after a negative bronchoscopy in paraesophageal lung tumors. We also evaluated the results EUS-FNA addressed to possible metastatic sites of these tumors (performed in the same session) and the EUS FNA related complications. The duration of the procedure and of the passage was measured.

\section{The EUS-FNA procedure}

All procedures were performed under anesthetic propofol deep sedation. A flexible EUS endoscope (Olympus GF-UCT180, Olympus ${ }^{\circledR}$ Medical Systems Europe, Ltd., Hamburg, Germany) was used. The echoendoscope was advanced into the duodenum and the patients were examined according to a validated and systematic published method for lung cancer patients, analyzing the 6 landmarks (liver, left adrenal gland, aorta, lymph stations 7, 4R, 4L) [20]. After the lung tumor evaluation in gray scale, color Doppler examination was performed in order to assess the vascularization of the tumor, and the vessels found to be interposed between the esophagus and the tumor. We performed also strain elastography in order to analyze the rigidity of the tumors compared to the surrounding tissues. FNAs were performed using $22 \mathrm{G}$ needles (Olympus EZ Shot $3+\circledR$, Olympus Medical Systems Europe, Ltd., Hamburg, Germany, or Boston Scientific Expect ${ }^{\circledR}$ needle) under ultrasonic guidance; the stylet was removed using the slow pull technique or suction method. After each FNA passage, we analyzed the aspirated specimens macroscopically, deciding if the samples were satisfactory (at least $20 \mathrm{~mm}$ length), or if another passage was needed. In cases with suspected metastatic lesions in other EUS-FNA accessible sites, we performed the biopsies in the same sessions.

Rapid on-site evaluation (ROSE) was not available. All procedures were performed by a single operator (RC), with more than 20 years of experience in endoscopy and more than 15 years in lung cancer percutaneous US-guided biopsy.

\section{Statistical analysis}

Nonparametric data are presented by mean and range. The diagnostic yield was calculated as the number of biopsies that resulted in a diagnosis divided by the total number of biopsies performed. 


\section{Results}

The analysis included 19 patients (15 males, mean age 65.1 years, range 45-80 years). Demographic data of the patients, tumor localization and dimension, number of passages and histological results are detailed in Table I.

The median tumors diameter was $6.7 \mathrm{~cm}$ (range 3.1$11 \mathrm{~cm}$ ) and a mean 2.1 passages/patient (1-3 passages) were performed.

In the same sessions, supplementary FNA was realized from the left adrenal glands in 2 patients (no. 4, 15), mediastinal lymph nodes in 3 patients (no. 4,10,19) and suspected liver metastasis in 2 patients (no.10, 18). The results revealed the same type of tumor as in the primary biopsied sites, confirming the metastatic lesions.

In all cases, lung EUS-FNA was diagnostic (19/19, 100\%).

No early or late complications following the EUSFNA procedures were registered.

The mean duration of the EUS-FNA procedure was 15 minutes and 13 seconds, and the mean passage time was 55 seconds.

In figure 1 we illustrate 2 cases included in the study.

Table I. Demographic data, tumor characteristics and pathology results

\begin{tabular}{lllllll}
\hline No. & Sex & Age & Localization & Dimension - cm (EUS) & No. of passages & Pathology results \\
\hline 1 & M & 74 & URL & 4.5 & 3 & Squamous cell carcinoma \\
2 & M & 67 & URL & 7 & 3 & Adenocarcinoma \\
3 & M & 61 & URL & 11 & 1 & Adenocarcinoma \\
4 & M & 76 & ULL & 6.2 & 3 & Adenocarcinoma \\
5 & M & 71 & LLL & 4 & 3 & Adenocarcinoma \\
6 & M & 78 & URL & 8 & 2 & Squamous cell carcinoma \\
7 & F & 57 & URL & 6 & 3 & Small cell neuroendocrine carcinoma \\
8 & M & 73 & ULL & 11 & 2 & Squamous cell carcinoma \\
9 & F & 57 & LRL & 8.2 & 2 & Adenocarcinoma \\
10 & M & 80 & URL & 7.4 & 3 & Squamous cell carcinoma \\
11 & M & 60 & LLL & 8 & 2 & Adenocarcinoma \\
12 & F & 73 & ULL & 5.4 & 2 & Small cell neuroendocrine carcinoma \\
13 & M & 62 & ULL & 6.2 & 2 & NSCLC \\
14 & F & 59 & URL & 3.5 & 2 & Breast carcinoma metastases \\
15 & M & 62 & ULL & 4.2 & 2 & Adenocarcinoma \\
16 & M & 67 & LRL & 3.1 & 2 & NSCLC \\
17 & M & 57 & ULL & 7.3 & 1 & Squamous cell carcinoma \\
18 & M & 58 & LLL & 7 & 1 & Squamous cell carcinoma \\
19 & M & 45 & ULL & 9.1 & 2 & Adenocarcinoma \\
\hline
\end{tabular}

$\mathrm{URL}=$ upper right lobe; ULL $=$ upper left lobe; $\mathrm{LRL}=$ lower right lobe; $\mathrm{LLL}=$ lower left lobe; NSCLC $=$ non-small cell lung carcinoma.

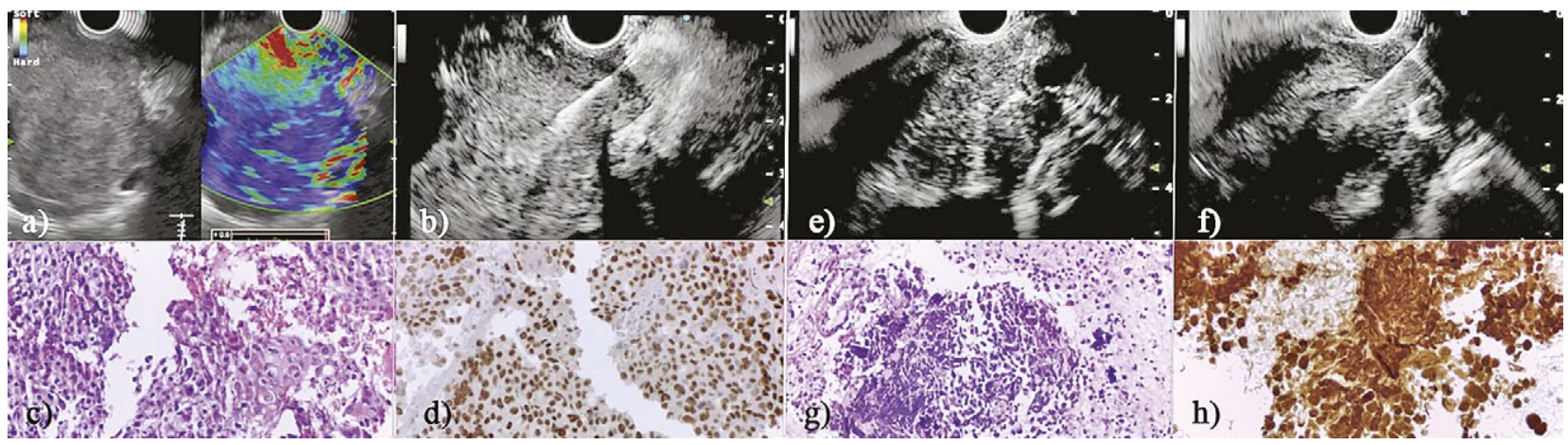

Fig 1. Case 1: a) EUS image of squamous cell lung cancer as a hypoechoic mass with an irregular contour, hard in comparison to the surrounding tissue in the elastography mode; b) EUS FNA of the squamous cell lung cancer - with the needle inside the tumor; c) Histopathological aspect of squamous cell carcinoma, HE stain (x 40); d). Squamous cell carcinoma - immunohistochemistry. Tumor cells are positive for p63 (x40). Case 2: e) Paraesophageally located small cell lung cancer - hypoechoic, irregular tumor, contiguous with mediastinal lymph nodes metastasis; f) EUS FNA of small cell lung cancer - 22G needle puncturing the tumor; g) Histopathology of small cell neuroendocrine carcinoma, HE stain (x 20); h) Small cell neuroendocrine carcinoma - immunohistochemistry. Tumor cells are positive for TTF-1 (x 40). 


\section{Discussion}

We analyzed the diagnostic yield of EUS-FNA of the paraesophageally located lung tumors and obtained an excellent rate, without complications. Also, we were able to diagnose, in the same EUS session, suspected metastatic lesions from other sites. The average duration of one passage was less than one minute and the duration of the whole procedure was 15 minutes.

The good sensitivity of the EUS-FNA for lung cancer staging, as well as mediastinal lymph nodes metastasis and the diagnosis of paraesophageally located lung tumors has already been reported [21]. EUS should be used along EBUS for the mediastinal staging in lung cancer, according to the latest guideline of the European Society of Gastrointestinal Endoscopy (ESGE), the European Respiratory Society (ERS) and the European Society of Thoracic Surgeons (ESTS) [4]. By using both techniques, mediastinal staging accuracy increases in cases shown as normal by a CT examination.

In this study we evaluated a series of patients with paraesophageal lung tumors that could not be histologically diagnosed throughout bronchoscopy or percutaneous US guided biopsy. The approach and puncture of the tumor by transesophageal route were considered feasible after the analysis of the CT images. There are few reported series of patients who have underwent EUS-FNA for this less common indication [21].

We obtained $100 \%$ sensitivity and diagnostic yield for the diagnosis of paraesophageally located lung cancers by EUS-FNA. Our data are concordant with the results of other studies supporting the importance of using EUS in these cases. A study involving pneumologists showed a very good diagnostic yield (26 of 27 patients) for EUS performed with an echobronhoscope [12]. A relatively recent systematic review and metaanalysis [21] which included 11 studies, with a total of 313 patients [9,13,22-24], showed an average diagnostic yield of 0.90 and an average sensitivity of 0.92 for diagnosing intrapulmonary lung tumors by EUS using the gastrointestinal echoendoscope. The complications rate found was less than $2 \%$ [21]. In our study, we had no complications either. Therefore, we consider that a proper indication for lung EUS-FNA is essential for a higher accuracy in this delicate area of lung pathology.

In our study, among the primary lung tumors, the proportion of the patients with non-small cell lung carcinoma (NSCLC) was $88.88 \%$, the other being small cell lung carcinoma, similar to the known statistical rates. We were also able to characterize the NSCLC as squamous and adenocarcinomas types, except for two cases in which the histological differentiation between the 2 types of NSCLC could not be done.

Another potential advantage of EUS in lung cancer is represented by the possibility to evaluate and procure samples from metastatic lesions - from liver, adrenal glands (mostly left side) abdominal lymph nodes or other sites, during the same session. We performed supplementary biopsies in 7 cases, all lesions being confirmed as metastasis. This feature increases the added value of EUS-FNA in the diagnosis and staging of paraesophageal lung cancer, particularly in cases where metastases are suspected and/or their imaging aspect is non-specific.

A limit of our study is represented by the relatively small number of patients included. Also, we did not include patients with lung tumors without contact to paraesophageal mediastinal pleura.

Despite the multiple advantages over other radiation techniques, EUS-FNA is still an underused technique for paraesophageally located lung tumors and for accessible metastatic sites. Efforts should be taken to extend knowledge concerning EUS-FNA.

\section{Conclusions}

EUS-FNA of the paraesophageally located lung tumors represents a very accurate and minimally invasive diagnostic method. The complication rate of this procedure is also very low.

\section{Conflicts of interest: none.}

\section{References}

1. Global Burden of Disease Cancer Collaboration, Fitzmaurice $\mathrm{C}$, Allen $\mathrm{C}$, et al. Global, regional, and national cancer incidence, mortality, years of life lost, years lived with disability, and disability-adjusted life-years for 32 cancer groups, 1990 to 2015: a systematic analysis for the global burden of disease study. JAMA Oncol 2017;3:524-548.

2. Rivera MP, Mehta AC, Wahidi MM. Establishing the diagnosis of lung cancer: diagnosis and management of lung cancer, 3rd ed: American College of Chest Physicians evidence-based clinical practice guidelines. Chest 2013;143(5 suppl):e142S-e165S.

3. Boskovic T, Stanic J, Pena-Karan S, et al. Pneumothorax after transthoracic needle biopsy of lung lesions under CT guidance. J Thorac Dis 2014;6 Suppl 1:S99-S107.

4. Vilmann P, Clementsen PF, Colella S, et al. Combined endobronchial and oesophageal endosonography for the diagnosis and staging of lung cancer. European Society of Gastrointestinal Endoscopy (ESGE) Guideline, in cooperation with the European Respiratory Society (ERS) and the European Society of Thoracic Surgeons (ESTS). Eur Respir J 2015;46:40-60. 
5. Heerink WJ, de Bock GH, de Jonge GJ, Groen HJ, Vliegenthart R, Oudkerk M. Complication rates of CTguided transthoracic lung biopsy: meta-analysis. Eur Radiol 2017;27:138-148.

6. Wallace MJ, Krishnamurthy S, Broemeling LD, et al. CTguided percutaneous fine-needle aspiration biopsy of small ( $\geq 1$ - cm) pulmonary lesions. Radiology 2002;225:823-828.

7. Kazerooni EA, Lim FT, Mikhail A, Martinez FJ. Risk of pneumothorax in CT-guided transthoracic needle aspiration biopsy of the lung. Radiology1996;198:371-375.

8. Ohno Y, Hatabu H, Takenaka D, et al. CT-guided transthoracic needle aspiration biopsy of small $(\leq 20 \mathrm{~mm})$ solitary pulmonary nodules. AJR Am J Roentgenol 2003;180:16651669.

9. Varadarajulu S, Hoffman BJ, Hawes RH, Eloubeidi MA. EUS-guided FNA of lung masses adjacent to or abutting the esophagus after unrevealing CT-guided biopsy or bronchoscopy. Gastrointest Endosc 2004;60:293-297.

10. Annema JT, Veseliç M, Rabe KF. EUS-guided FNA of centrally located lung tumors following a non-diagnostic bronchoscopy. Lung Cancer 2005;48:357-361.

11. Araya T, Demura Y, Kasahara K, et al. Usefulness of transesophageal bronchoscopic ultrasound-guided fineneedle aspiration in the pathologic and molecular diagnosis of lung cancer lesions adjacent to the esophagus. J Bronchology Interv Pulmonol 2013;20:121-126.

12. Steinfort DP, Farmer MW, Irving LB, Jennings BR. Pulmonologist-performed per-esophageal needle aspiration of parenchymal lung lesions using an EBUS bronchoscope: diagnostic utility and safety. J Bronchology Interv Pulmonol 2017;24:117-124.

13. Sawhney MS, Kratzke RA, LederleFA, HolmstromAM, Nelson DB, Kelly RF. EUS-guided FNA for the diagnosis of advanced lung cancer. Gastrointest Endosc 2006;63:959-965.

14. Songür N, Songür Y, Bircan S, Kapucuoğlu N. Comparison of 19- and 22-gauge needles in EUS-guided fine needle aspiration in patients with mediastinal masses and lymph nodes. Turk J Gastroenterol 2011;22:472-478.
15. Vazquez-Sequeiros E, Levy MJ, Van Domselaar M, et al. Diagnostic yield and safety of endoscopic ultrasound guided fine needle aspiration of central mediastinal lung masses. Diagn Ther Endosc 2013;2013:150492.

16. Nasir BS, Edwards M, Tiffault V, et al. Transesophageal pulmonary nodule biopsy using endoscopic ultrasonography. J Thorac Cardiovasc Surg 2014;148:850-855.

17. Skovgaard Christiansen I, Kuijvenhoven JC, Bodtger U, et al. Endoscopic ultrasound with bronchoscope-guided fine needle aspiration for the diagnosis of paraesophageally located lung lesions. Respiration 2019;97:277-283.

18. Colella S, Vilmann P, Konge L, Clementsen PF. Endoscopic ultrasound in the diagnosis and staging of lung cancer. Endosc Ultrasound 2014;3:205-212.

19. Uemura S, Yasuda I, Kato T, et al. Preoperative routine evaluation of bilateral adrenal glands by endoscopic ultrasound and fine needle aspiration in patients with potentially resectable lung cancer. Endoscopy 2013;45:195-201.

20. Konge L, Vilmann P, Clementsen P, Annema JT, Ringsted C. Reliable and valid assessment of competence in endoscopic ultrasonography and fine-needle aspiration for mediastinal staging of non-small cell lung cancer. Endoscopy 2012;44:928-933.

21. Korevaar DA, Colella S, Spijker R, et al. Esophageal endosonography for the diagnosis of intrapulmonary tumors: a systematic review and meta-analysis. Respiration 2017;93:126-137.

22. Paquin SC, Hoffman BJ, Hawes RH, Chong AK, Faias SR, Hoda RS. Utility of on-site cytologic assessment of transesophageal endoscopic ultrasound-guided fine needle aspiration of lung masses (abstract). Gastrointest Endosc 2005;61:AB296.

23. Hernandez A, Kahaleh M, Olazagasti J, et al. EUS-FNA as the initial diagnostic modality in centrally located primary lung cancers. J Clin Gastroenterol 2007;41:657-660.

24. Assisi D, Filippetti M, Federici T, Visca P, Anti M. Role of EUS FNA in diagnosis and staging of lung cancer. Dig Liver Dis 2013;45 Suppl 2:S76-S77. 